\title{
Magnetic friction of a nanometer-sized tip scanning a magnetic surface: Dynamics of a classical spin system with direct exchange and dipolar interactions between the spins
}

\author{
C. Fusco and D. E. Wolf \\ Department of Physics and CeNIDE, University of Duisburg-Essen, D-47048 Duisburg, Germany \\ U. Nowak \\ Department of Physics, University of York, York 105DD, United Kingdom
}

\begin{abstract}
We theoretically study the occurrence of magnetic friction of a nanometer-sized tip scanning a magnetic surface by studying the dynamics of a model of classical spins interacting through dipolar and exchange interactions, neglecting thermal effects. We find that for small scanning velocities, the friction force linearly scales with the velocity, with a slope proportional to the phenomenological damping parameter of the LandauLifshitz-Gilbert equation. At higher velocities, the friction vs velocity relationship becomes rather complex with the presence of a maximum that is explained by the excitations of spin-wave resonances in the sample.
\end{abstract}

PACS number(s): 75.70.Rf, 68.35.Af, 68.37.Rt, 07.79.Pk

\section{INTRODUCTION}

Understanding the microscopic mechanisms of friction between solid surfaces is an important issue both from a fundamental point of view as well as for practical applications. ${ }^{1-3}$ When the size of the contacting surfaces under investigation is reduced to the nanometer scale, many novel physical effects occur. ${ }^{3}$ The field of nanoscale friction has experienced an enormous advancement in the last two decades thanks to the introduction and development of the atomic force microscopy (AFM) technique, ${ }^{2,4}$ This technique, which basically measures the forces between a moving tip and a sample surface with atomic resolution, has made it possible to identify different channels of energy dissipation, e.g., phononic and electronic friction. While a considerable amount of work has been done for nonmagnetic materials, the mechanisms leading to friction associated with magnetic interactions between magnetic surfaces have not been studied up to now. Magnetic materials are now controllable down to the nanometer scale, leading to a broad interest in the understanding of the magnetism of small structures and particles. ${ }^{5}$ Magnetic force microscopy (MFM), where a magnetic tip is scanned over a magnetic surface, has been mainly employed to investigate surface magnetism and to visualize domains with nanometer-scale resolution. ${ }^{6} \mathrm{Al}-$ though recent studies attempted to measure the energy dissipation between an oscillating tip and a magnetic sample, ${ }^{7,8}$ the dependence of the friction force on the sliding velocity of a scanning tip has not been considered yet. The relative motion of the tip with respect to the surface can lead to the creation of spin waves, which dissipate energy, giving rise to magnetic friction.

The aim of the present work is to investigate the magnetic friction between a moving tip and a magnetic surface by studying the dynamics of a model of classical magnetic moments localized on a lattice. In an attempt to keep the model as simple as possible, we idealize the tip as a single point dipole, thereby neglecting any dissipation within the tip and focusing solely on the physics of the friction in the substrate. In Sec. II, we define our model and simulation methods and we describe how to calculate the magnetic friction force. In Sec. III, we present our results on the dependence of the magnetic friction on the scanning velocity in the absence of thermal effects. Some concluding remarks are given in Sec. IV.

\section{MODEL AND SIMULATION TECHNIQUES}

We consider a simple cubic lattice with normalized classical magnetic moments $\mathbf{S}_{\mathbf{i}}(i=1, \ldots N)$ as dynamic variables. The system size is $L_{x} \times L_{y} \times L_{z}$ and open boundary conditions are used. A tip with a fixed magnetic moment $\mathbf{S}_{\text {tip }}$ moves with fixed velocity $v_{\text {tip }}$ in the $x$ direction across the $x y$ surface of the substrate. The Hamiltonian of the total system (tip+substrate) is the sum of the Hamiltonian describing the interactions between the spins in the substrate $\left(H_{\text {sub }}\right)$ and the Hamiltonian corresponding to the interaction between the tip spin and the spins in the substrate $\left(H_{\text {tip-sub }}\right)$,

$$
H=H_{\text {sub }}+H_{\text {tip-sub }}
$$

The Hamiltonian of the substrate is that of a classical spin model,

$$
H_{\text {sub }}=-J \sum_{\langle i j} \mathbf{S}_{i} \cdot \mathbf{S}_{j}-w \sum_{i<j} \frac{3\left(\mathbf{S}_{i} \cdot \mathbf{e}_{i j}\right)\left(\mathbf{S}_{j} \cdot \mathbf{e}_{i j}\right)-\mathbf{S}_{i} \cdot \mathbf{S}_{j}}{r_{i j}^{3}}
$$

where $r_{i j}=\left|\mathbf{r}_{i}-\mathbf{r}_{j}\right|, \mathbf{r}_{i}$ is the position of the spin at site $i$ (in units of the lattice constant $a$ ), $\mathbf{e}_{i j}$ are unit vectors in the direction of $\mathbf{r}_{i j}, J$ is the exchange constant, and $(i j)$ denotes that the sum is performed only on the nearest neighbors. The tip-substrate interaction is of purely dipolar nature,

$$
H_{\text {tip-sub }}=-w \sum_{i} \frac{3\left(\mathbf{S}_{i} \cdot \mathbf{e}_{i \mathrm{tip}}\right)\left(\mathbf{S}_{\mathrm{tip}} \cdot \mathbf{e}_{\mathrm{itip}}\right)-\mathbf{S}_{i} \cdot \mathbf{S}_{\mathrm{tip}}}{r_{\text {itip }}^{3}}
$$

where $r_{i \text { tip }}=\left|\mathbf{r}_{i}-\mathbf{r}_{\text {tip }}\right|, \mathbf{r}_{\text {tip }}$ being the tip position and $\mathbf{e}_{i \text { ip }}$ $=\mathbf{r}_{\text {itip }} / r_{\text {itip }}$. The spin motion is described by the LandauLifschitz-Gilbert (LLG) equation, ${ }^{9}$ 


$$
\frac{\partial \mathbf{S}_{i}}{\partial t}=-\frac{\gamma}{\left(1+\alpha^{2}\right) \mu_{s}} \mathbf{S}_{i} \times\left[\mathbf{h}_{i}(t)+\alpha \mathbf{S}_{i} \times \mathbf{h}_{i}(t)\right] .
$$

In Eq. (4), $\gamma=1.76 \times 10^{11}(\mathrm{Ts})^{-1}$ is the gyromagnetic ratio, $\mu_{s}$ is the absolute value of the magnetic moment, $\alpha$ is the dimensionless damping constant $(0 \leq \alpha \leq 1)$, and $\mathbf{h}_{i}(t)$ is the effective field

$$
\mathbf{h}_{i}(t)=-\frac{\partial H}{\partial \mathbf{S}_{i}} .
$$

The first part of Eq. (4) describes the spin precession while the second part includes the relaxation of the spins in a phenomenological way. The LLG [Eq. (4)] without damping $(\alpha=0)$ is the classical limit of the Heisenberg equation for the precessional motion of the electron spin operator. The damping term is added in a phenomenological manner according to the work of Gilbert, based on the Gilbert equation,

$$
\frac{\partial \mathbf{S}_{i}}{\partial t}=-\frac{\gamma}{\mu_{s}} \mathbf{S}_{i} \times \mathbf{h}_{i}+\alpha \mathbf{S}_{i} \times \frac{\partial \mathbf{S}_{i}}{\partial t} .
$$

It is easy to prove that the two formulations, the LLG [Eq. (4)] and the Gilbert [Eq. (6)] are equivalent. ${ }^{10}$ Alternatively, the Gilbert equation can be derived from a variational principle using a combination of a Lagrangian and a Rayleigh function. Our goal is not to investigate or improve the Gi1bert damping itself but we rather assume that this equation is valid and then we derive a way to numerically calculate the dissipation. For the readers who are interested in the fundamentals of Gilbert damping, we refer them to Refs. 10 and 11.

In order to calculate the magnetic friction force $F_{\text {fric }}$ experienced by the moving tip, we need to find the expression of the rate of energy dissipation in the system, $P_{\text {diss }}$. The total energy rate is given by

$$
\frac{d H}{d t}=\frac{\partial H}{\partial t}+\sum_{i=1}^{N} \frac{\partial H}{\partial \mathbf{S}_{i}} \cdot \frac{\partial \mathbf{S}_{i}}{\partial t} .
$$

The first term on the right-hand side of Eq. (7) is due to the effect of the moving tip spin, which produces an explicit time dependence of the Hamiltonian,

$$
\frac{\partial H}{\partial t}=\frac{\partial H}{\partial \mathbf{r}_{\text {tip }}} \cdot \mathbf{v}_{\text {tip }} \text {. }
$$

In order to identify the rate of dissipated energy in Eq. (7), it is convenient to use Eq. (6). Then Eq. (7) can be rewritten as

$$
\frac{d H}{d t}=\frac{\partial H}{\partial \mathbf{r}_{\text {tip }}} \cdot \mathbf{v}_{\text {tip }}+\alpha \sum_{i=1}^{N} \frac{\partial H}{\partial \mathbf{S}_{i}} \cdot\left(\mathbf{S}_{i} \times \frac{\partial \mathbf{S}_{i}}{\partial t}\right) .
$$

In the steady state, the total energy rate is zero on the average so that all of the energy pumped into the system is eventually dissipated, i.e.,

$$
\left\langle\frac{d H}{d t}\right\rangle=\left\langle P_{\text {pump }}\right\rangle-\left\langle P_{\text {diss }}\right\rangle=0
$$

Then the pumping power is given by

$$
\left\langle P_{\text {pump }}\right\rangle=\left\langle\frac{\partial H}{\partial \mathbf{r}_{\text {tip }}} \cdot \mathbf{v}_{\text {tip }}\right\rangle,
$$

and the average rate of dissipated energy is

$$
\left\langle P_{\text {diss }}\right\rangle=-\alpha\left\langle\sum_{i=1}^{N} \frac{\partial H}{\partial \mathbf{S}_{i}} \cdot\left(\mathbf{S}_{i} \times \frac{\partial \mathbf{S}_{i}}{\partial t}\right)\right\rangle .
$$

As expressed by Eqs. (11) and (12), the energy pumped into the system comes from the energy transferred from the tip to the substrate while moving, while the dissipated energy is proportional to the damping constant $\alpha$ and to the spin precession velocity. The friction force $F_{\text {fric }}$ is simply obtained as

$$
F_{\text {fric }}=\left\langle P_{\text {diss }}\right\rangle / v_{\text {tip }} \text {. }
$$

We have numerically integrated the LLG Gilbert [Eq. (4)] by using the Heun scheme. ${ }^{12}$ The initial configuration is prepared in the following way. The tip spin, pointing in the $z$ direction, is placed in a position $\mathbf{r}_{\text {tip }}^{(\text {in) }}=\left[x_{\text {tip }}^{(\text {in) }}, L_{y} / 2,1\right]$ above the substrate, which is ferromagnetically ordered in the $x$ direction. Then the substrate spins are allowed to relax for fixed tip until they have reached a stationary state, which is taken as the initial configuration for the simulation. In this state, the spin components $S_{i x}$ on opposite sides of the tip have opposite sign, reflecting their adjustment to the dipole field of the tip. Then the tip starts moving with constant velocity $v_{\text {tip }}$ in the $x$ direction until it reaches a prescribed position $\mathbf{r}_{\text {tip }}^{(\text {fin })}=\left[x_{\text {tip }}^{(f i n)}, L_{y} / 2,1\right]$. In order to avoid boundary effects, we choose $x_{\text {tip }}^{(\mathrm{in})}=L_{x} / 6$ and $x_{\text {tip }}^{(\mathrm{fin})}=5 L_{x} / 6$, so that the effective distance traveled by the tip is $\Delta x_{\text {tip }}=2 L_{x} / 3$. The average $\langle\cdots\rangle$ in $\mathrm{Eq} .(13)$ is calculated as a time average after having reached a steady state.

For convenience, the equation of motion [Eq. (4)] can be rewritten in rescaled time and energy units. In fact, we can rescale the dipolar interaction parameter as

$$
w^{\prime}=w / J
$$

and the time as

$$
t^{\prime}=t J \gamma / \mu_{s} .
$$

The lengths appearing in the Hamiltonian are already ex pressed in the units of the lattice constant $a$. Rescaled scanning velocity and friction force are

$$
v_{\text {tip }}^{\prime}=\frac{\mu_{s}}{\gamma J a} v_{\text {tip }}
$$

and

$$
F_{\text {fric }}^{\prime}=\frac{a}{J} F_{\text {fric }},
$$

respectively. We used the following rescaled parameters in the simulations (we omit the ' from now on for simplicity): $w=0.01, \mathbf{S}_{\mathrm{tip}}=(0,0$, and 1000$)$. The magnetic moment of the whole tip is modeled as a single strong dipole close to the surface in order to produce a large and sufficiently localized effective perturbation suitable for a numerical investigation. As for the damping constant $\alpha$, we have used different values ranging from 0.1 to 0.5 . The data presented in the following 

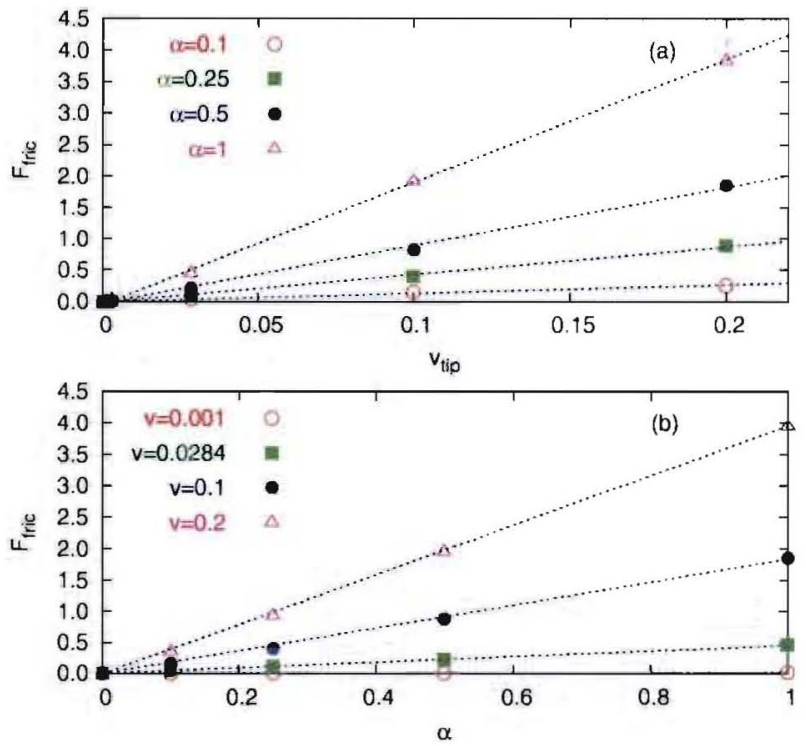

FIG. I. (Color online) (a) Behavior of magnetic friction $F_{\text {fric }}$ as a function of the tip scanning velocity $v_{\text {tip }}$ for different values of the damping constant and (b) as a function of the damping constant $\alpha$ for different values of the tip scanning velocity. The symbols are the numerical results while the lines are linear fits to the points. Quantities are reduced according to Eqs. (14)-(17).

were obtained for system sizes $\left(L_{x}, L_{y}, L_{z}\right)=\left(240,10, L_{z}\right)$, with $L_{z}$ between 1 and 6 .

\section{VELOCITY DEPENDENCE OF MAGNETIC FRICTION}

Following the procedure outlined in Sec. II, we calculated for each velocity $v_{\text {tip }}$ the friction force between the tip and the substrate by using Eqs. (13) and (12). The dependence of $F_{\text {fric }}$ on $v_{\text {tip }}$ is strongly sensitive to the velocity range investigated. We will first consider the case of small scanning velocities, which is of interest in AFM experiments, and then we will focus on the behavior of the friction force for wider ranges of scanning velocities.

\section{A. Small scanning velocities}

In Fig. 1(a), we show the behavior of the friction force $F_{\text {fric }}$ as a function of the tip velocity $v_{\text {tip }}$ for different values of the damping constant $\alpha$ at relatively small values of the tip velocity.

It is clear from Fig. I that our data are consistent with a linear relationship between $F_{\text {fric }}$ and $v_{\text {tip }}$. The origin of this behavior could be traced back to the form of the phenomenological dissipation term introduced in the LLG equation, which is proportional to the spin velocity $\frac{t \boldsymbol{S}_{i}}{\partial t}$ and thus acts as a sort of viscous friction. Furthermore, our results presented in Fig. 1(b) show a linear dependence of the friction force on the damping parameter at such small velocities, which follows directly from Eq. (12) and reflects the form of the damping introduced in Eq. (4). This is analogous to what happens in a mechanical model of friction, e.g., the Tomlinson model, where one introduces a dissipation term propor-
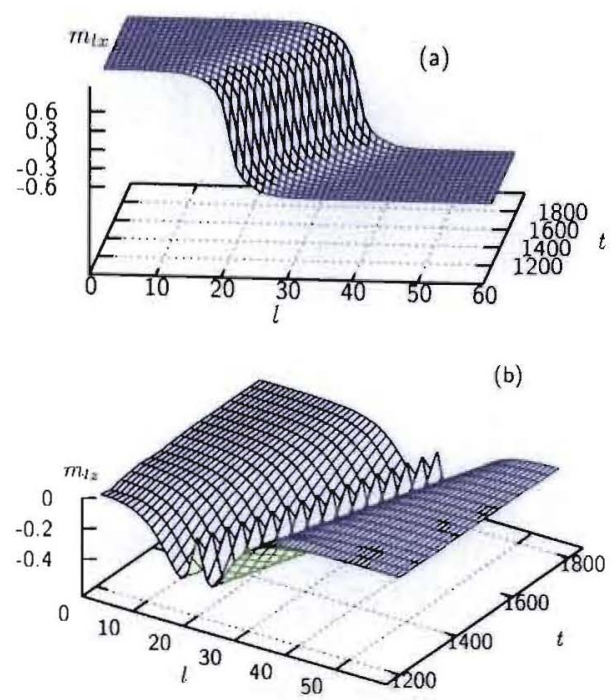

FIG. 2. (Color online) $x$ and $z$ components, (a) and (b), respectively, of the layer magnetization as a function of the layer index $l$ and time $t$. The velocity of the tip is $v_{\text {tip }}=0.0284$ and $\alpha=0.1$. Quantities are reduced according to Eqs. (14)-(17).

tional to the atom velocity and recovers a linear dependence of friction on macroscopic velocity with a slope proportional to the damping parameter. ${ }^{13}$

In order to have a physical estimate of the order of magnitude of our scanning velocitics, we notice that velocities are rescaled according to Eq. (I6) and that typical values of our parameters are $J \simeq 10^{-21} \mathrm{~J}, a \simeq 10^{-10} \mathrm{~m}$, and $\mu_{s}$ $\approx 10^{-23} \mathrm{~J} / \mathrm{T}$. This means that our smallest velocity $v_{\text {tip }}^{\prime}$ $=10^{-3}$ in Fig. I(a) would correspond to $v_{\text {tip }} \simeq 1 \mathrm{~m} / \mathrm{s}$. This velocity is certainly much higher than typical scanning velocities used in MFM. On the other hand, it is much lower than the velocity of a read/write head on a spinning magnctic disk in a commercial storage device.

Magnetic friction essentially results from the creation of spin waves due to the relative motion between the tip and the substrate. In order to get a deeper insight into the mechanisms of magnetic energy dissipation, we consider the layer magnetization $\mathbf{m}_{/}$, defined as an average over all magnetic moments in a $y z$ plane at a fixed value of the $x$ coordinate (equal to the layer index $l$ ). In Fig. 2, we display the $x$ and $z$ components of this layer magnetization. From this figure, it is clear that there is a front propagating in the system caused by the moving tip. The motion of this wave dissipates energy, resulting in a finite friction force. In particular, the bumps in $m_{l z}$ reflect the reorientation of the spins as soon as they are approached by the tip.

The spin waves propagate into the system until they are damped by the phenomenological damping term introduced in LLG [Eq. (4)]. Thus, the damping rate is proportional to the parameter $\alpha$. This effect is shown in Fig. 3, where we have displayed the friction force as a function of the tip velocity for systems with different values of $L_{z}$ and for two values of the damping $\alpha$. For the larger $\alpha$, the curves for $L_{z} \geq 2$ are practically indistinguishable, reflecting the fast damping of the spin excitations, while for the smaller $\alpha$ finite size effects are more evident, especially at larger velocities. 


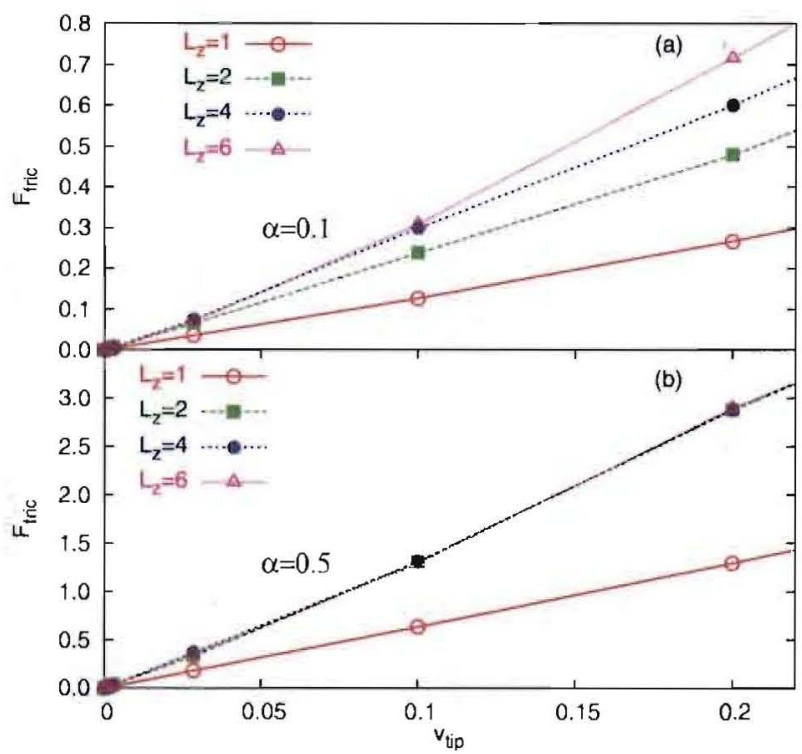

FIG. 3. (Color online) Magnetic friction as a function of tip velocity for $\alpha=0.1$ (a) and $\alpha=0.5$ (b), and different values of $L_{2}$ as indicated in the labels. Quantities are reduced according to Eqs. (14)-(17).

\section{B. Larger scanning velocities}

By increasing the tip velocity, the spin dynamics becomes much more complex. Figure 4 illustrates the complexity of the relation between magnetic friction and tip velocity for $v_{\text {tip }} \leq 10$. The values of $F_{\text {fric }}$ in Fig. 4 have been calculated using Eq. (13), averaging $P_{\text {diss }}$ over different time intervals depending on the value of $v_{\text {tip }}$ since the duration of the steady state region is generally shorter for higher values of $v_{\text {tip. }}$. After an initial increase in velocity, the magnetic friction reaches a maximum at a value $v_{\text {tip }}=v_{0}$, which slightly depends on $\alpha$ and then it decreases with a rate significantly slower than that of the increasing part. The value of the

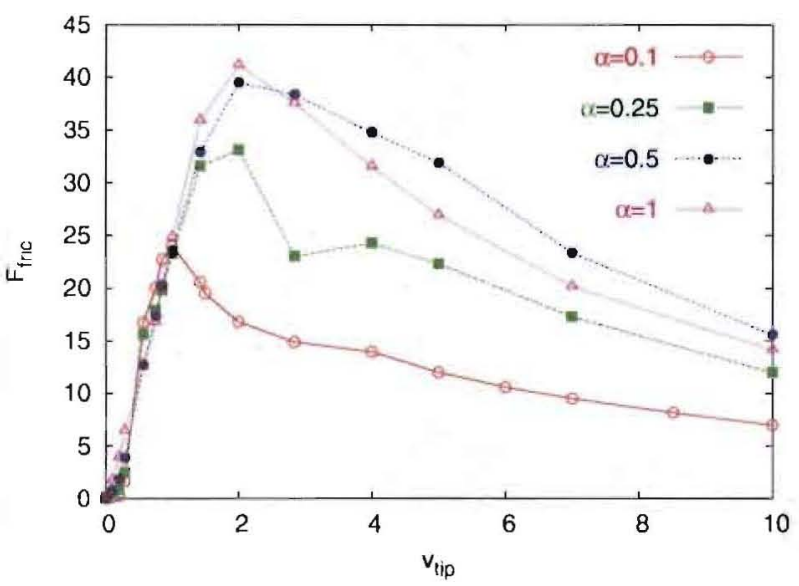

FIG. 4. (Color online) Magnetic friction as a function of tip velocity for different values of $\alpha$, as indicated by the labels, in a wide range of tip velocities. Quantities are reduced according to Eqs. (14)-(17).

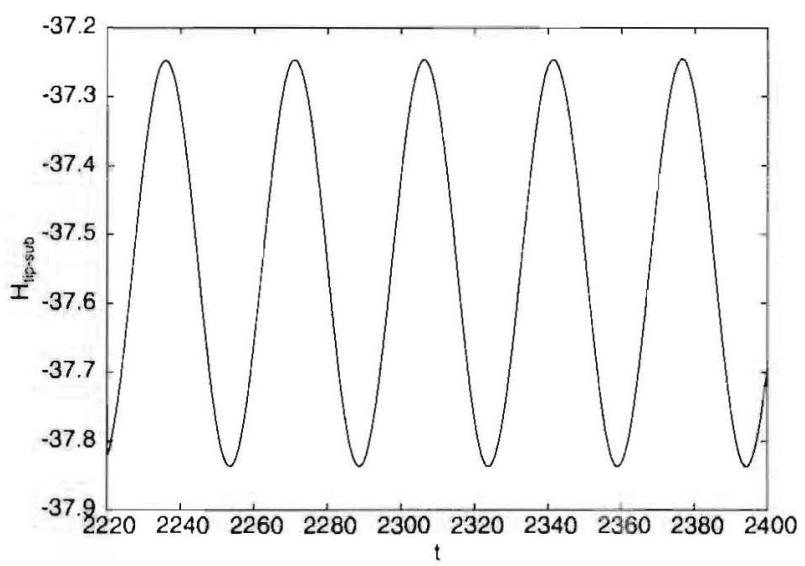

FIG. 5. Tip-substrate interaction as a function of time for $v_{\text {tip }}$ $=0.0284$ and $\alpha=1$. Notice that the period is in agreement with Eq. (18). Quantities are reduced according to Eqs. (14)-(17).

maximum is also sensitive to the magnitude $\alpha$.

We interpret this intriguing behavior as related to the spin waves excited in the sample by the moving tip. The tip, when passing over the surface of the sample, interacts with the periodic spin lattice. The resulting periodicity of the tipsubstrate interaction $H_{\text {tip-sub }}$ is shown in Fig. 5. This interaction leads to the excitation of spin waves with a spectrum that has maximum frequency defined by the tip velocity,

$$
\omega \approx \frac{2 \pi v_{\mathrm{tip}}}{a} .
$$

The dispersion relation for spin waves on a square lattice with nearest-neighbor interaction, which we consider here is $^{14}$

$$
\omega(q)=\frac{4 J \gamma}{\mu_{s}}[1-\cos (q a)] .
$$

The maximum spin wave frequency for $q=\pi / a$ is hence

$$
\omega_{\max }=\frac{8 J \gamma}{\mu_{s}} .
$$

Consequently, the highest energy excitation is for tip velocities (in physical units) of the order of

$$
v_{\max } \approx \frac{4 a J \gamma}{\pi \mu_{s}}
$$

where a velocity, in our rescaled units, is simply $v_{\max }^{\prime}=4 / \pi$. To have an idea of the order of magnitude of $v_{\max }$, with the same parameters given before, this corresponds to $v_{\max }$ $\simeq 10^{3} \mathrm{~m} / \mathrm{s}$. Above that tip velocity, spin waves with a frequency according to Eq. (18) can no longer be excited and the energy transfer from tip to the spin lattice becomes less efficient. Consequently, the dissipated power drops down and with that, the friction force. These considerations are in good in agreement with Fig. 4, where the maxima of the friction force are indeed around rescaled velocities of $v^{\prime} \approx 1$ although with some indication of an $\alpha$ dependence, which is not described by our arguments above. 
The excited spin waves have well-defined frequencies, the upper limit of which, according to Eq. (18), is proportional to the tip velocity. With that, the energy $E \sim \omega$ of the excited spin waves is also proportional to the velocity. The spin waves are locally excited by the tip but their energy is spread over the sample and dissipates due to the damping term in the equation of motion. The dissipated power (energy per time) in a stationary state is then proportional to $v^{2}$. Since $F_{\text {fric }}$ is given by $\mathrm{Eq}$. (13), we recover the linear behavior for $F_{\text {fric }}$ as a function of $v_{\text {tip }}$ at small values of $v_{\text {tip }}$, found in Fig. $1(\mathrm{a})$

\section{CONCLUSIONS AND PERSPECTIVES}

In this work, we have investigated for the first time the magnetic friction between a moving point dipole and a magnetic surface. Our analysis is based on the dynamics of a classical spin system following the Landau-Lifshitz-Gilbert equation with direct exchange and dipolar interactions between the spins. We find that the friction force is proportional to the phenomenological damping constant, as one would expect. Our main findings, however, are: (i) A linear dependence of the friction force on the scanning velocity for small velocities and (ii) the occurrence of a resonance at very high velocities, where the magnetic friction has a very high peak. Both of these results can be interpreted in terms of excitations of spin waves in the substrate. In order to focus on this new dissipation mechanism, we discarded the magnetic degrees of freedom of the scanning tip in our model. The price we pay for this is that a realistic estimate of the strength of the effect for a MFM or for a hard disk, where the read/write head flies over the magnetic substrate, becomes impossible. From Fig. 1 and Eq. (17), it turns out that the magnetic friction force in our simulation is rather weak (of the order of $10^{-12} \mathrm{~N}$ only), in spite of the large magnetic moment of the tip and its small distance from the surface. The important message here is that there is a magnetic contribution to friction even at $T=0$. Whether its value may be enhanced at larger temperature, by different spin structures (like antiferromagnetic ordering), by a more realistic modeling of the tip, and last but not the least, by a larger spin system, providing more phase space to absorb energy, remains to be seen. Certainly, suitable experiments on magnetic friction are encouraged.

It is worth noting that the velocity dependence of magnetic friction could be influenced by the dynamical treatment of the spin system and by the specific form of the dissipation term. For instance, in a recent Monte Carlo study on the magnetic friction of the Ising model, it was found that the friction force is independent of velocity, ${ }^{15}$ in contrast to our findings. Our analysis is based on the dynamics of the LLG equations with a form of the dissipative term, which is purely phenomenological. An analysis of different kinds of damping terms might lead to deviating results. We hope that our work stimulates further theoretical and experimental investigations of the microscopic origin of magnetic friction.

\section{ACKNOWLEDGMENTS}

This work was supported by the German Research Society (DFG) within SFB 616 "Energy Dissipation at Surfaces" and by the Alexander von Humboldt foundation.
${ }^{1}$ See, for example, B. N. J. Persson, Stiding Friction: Physical Principles and Applications (Springer, Berlin, 1998).

${ }^{2}$ E. Gnecco, R. Bennewitz, T. Gyalog, and E. Meyer, J. Phys: Condens. Matter 13, R619 (2001).

${ }^{3}$ M. Urbakh, J. Klafter, D. Gourdon, and J. Israelachvili, Nature (London) 430, 525 (2004).

${ }^{4}$ R. W. Carpick and M. Salmeron, Chem. Rev. (Washington, D.C.) 97, 1163 (1997).

${ }^{5}$ F. J. Himpsel, J. E. Ortega, G. J. Mankey, and R. F. Willis, Adv, Phys. 47, 511 (1998).

${ }^{6}$ Y. Martin and H. K. Wickramasinghe, Appl. Phys. Lett. 50, 1455 (1987)

${ }^{7}$ P. Grütter, Appl. Phys. Lett. 71, 279 (1997).
${ }^{8}$ Y. Liu and P. Grütter, J. Appl. Phys. 83, 5922 (1998).

${ }^{9}$ W. F. Brown, Phys. Rev. 130, 1677 (1963).

${ }^{10}$ W. F. Brown, Micromagnetics (Wiley, New York, 1963).

"I. Miltat, G. Albuquerque, and A. Thiaville, in Spin Dynamics in Confined Magnetic Structures, Topics in Applied Physics Vol. 83 , edited by $\mathrm{I}$. B. Hillebrands and $\mathrm{K}$. Ounadjela (SpringerVerlag, Berlin, 2002).

12 J. L. Garcia-Palacios and F. J. Lázaro, Phys. Rev. B 58, 14937 (1998).

${ }^{13}$ C. Fusco and A. Fasolino, Phys. Rev. B 71, 045413 (2005).

${ }^{14} \mathrm{D}$. C. Mattis, The Theory of Magnetism II (Springer Verlag, Heidelberg, 1986)

${ }^{15}$ D. Kadau and D. E. Wolf, arXiv:0706.3610 (unpublished) 